American Journal of Applied Sciences 7 (6): 807-810, 2010

ISSN 1546-9239

(C) 2010 Science Publications

\title{
Effects of Total Ionizing Dose on Bipolar Junction Transistor
}

\author{
${ }^{1}$ Chee Fuei Pien, ${ }^{1}$ Haider F. Abdul Amir, ${ }^{1}$ Saafie Salleh and ${ }^{2}$ Azali Muhammad \\ ${ }^{1}$ Physics with Electronics Program, School of Science and Technology, \\ University Malaysia Sabah, Locked Bag No. 2073, 88999 Kota Kinabalu, Sabah, Malaysia \\ ${ }^{2}$ Industry Technology Division, Malaysian Nuclear Agency, \\ Bangi, 43000 Kajang, Selangor, Malaysia
}

\begin{abstract}
Problem statement: The amount of ionizing radiation that Bipolar Junction Transistor (BJT) devices encounter during their lifecycle degrades both of their functional and electrical parameter performances. The different radiation environments either in space, high energy physics experiments, nuclear environment or fabrication process as well as for standard terrestrial operation possess an impact on the devices. Approach: In this research, analytical studies of the effects of ionizing radiation introduced in Commercial-Off-The Shelf (COTS) NPN BJTs by ${ }^{60} \mathrm{Co}$ gamma $(\gamma)$ rays had been performed. Results: It was observed that exposure of BJTs to ${ }^{60} \mathrm{Co}$ caused ionizing radiation damage. Ionizing radiation damage was caused mainly by excess charges trapped on or near the surfaces of their insulating layers and interfaces. This phenomenon reduced the minority carrier lifetime and thus, leading to a decrease in the current gain of the BJTs. Conclusion: This ionizing radiation effect was found to arouse either a permanent or temporarily damage in the devices depending on their current drives and also the Total Ionizing Dose (TID) absorbed. The performance and degradation of selected BJT devices during irradiation with respect to total dose ${ }^{60} \mathrm{Co}$ were presented in this study.
\end{abstract}

Key words: Bipolar Junction Transistor (BJT), gamma $(\gamma)$ rays, in situ, Total Ionizing Dose (TID)

\section{INTRODUCTION}

Depending on the type of semiconductor device and its deployment, it may be exposed to two types of radiation environments which are the exoatmospheric (space) and endoatmospheric (earth) radiation (Guitierrez, 1999). The radiation that a semiconductor device endured can alter or damage the normal functioning and resulting in critical system failure. The radiation effects on semiconductor devices can be categorized into four: Electromagnetic Pulse (EMP) effects, high dose rate effects, Single Event Upsets (SEU) and total dose effects (Aghara, 2003).

EMP encompasses phenomena in the electromagnetic spectrum from very low frequencies of less than a hertz to Ultrahigh Frequencies (UHF). This EMP phenomenon arises from the incidence of an electromagnetic wave on the semiconductor devices (Bhuva, 1987). The resulting electric and magnetic fields from EMP may interact with electrical and electronic systems to produce damaging current and voltage surges. This effect is temporary and the system can bounce back to normal operating condition as soon as the radiation pulse terminates.
Dose rate effects induce a temporary failure of circuit operation which lasts as long as the radiation pulse exists. These exposures are normally short and may last from a few nano-seconds (ns) to micro-seconds $(\mu \mathrm{s})$.

SEU or soft error is defined as radiation-induced errors in semiconductor devices caused when charged particles lose energy by ionizing the medium through which they pass, leaving behind a wake of electron-hole pairs. An SEU may occur in analog, digital, or optical components, or may have effects in surrounding interface circuitry (Messenger and Ash, 1992). This type of error is temporary and no permanent damage to the circuits.

Total dose effects, however, are permanent damage effects. The device parameters and performance are degraded permanently due to generation of interface states and trapped charged in the oxide when exposed to a total dose (Aghara, 2003). The work reported here primarily deals with this type of radiation damage due to TID.

The TID effects are cumulative and these effects are gradual which take place during the whole lifetime of the devices exposed to radiation. Depending on the

Corresponding Author: Chee Fuei Pien, Physics with Electronics Program, School of Science and Technology, University Malaysia Sabah, Locked Bag No. 2073, 88999 Kota Kinabalu, Sabah, Malaysia 
photon energy, $\mathrm{E}_{\mathrm{PH}}$, different fundamental interactions can take place, which result in the creation of an energetic free carrier (or pair) in the material (Li et al., 2007). This includes the photoelectric effect, Compton Effect and pair production (Claeys and Simoen, 2002).

In recent study, it was found that the generation and annealing characteristics of the oxide-trapped charges and the interface states in semiconductor devices due to TID are strongly affected by the gate bias polarity and electric field, the oxide thickness, the oxide process history, the temperature during irradiation and subsequent annealing, the silicon substrate orientation and also the total dose absorbed (Najim, 2009).

The increasing demands of non-hardened COTS components in viable space avionics systems, reactor control system and defense systems demand an adapted radiation hardness assurance management (Park, 2004). In particular, an optimization should be made on radiation specifications for ascertaining and reducing excessive margin. System-oriented studies should also be done on how radiation effects actually impact on current electronic components. This study will present the ionizing radiation effects on BJTs, the radiation response of Device Under Test (DUT) and radiation tolerance of DUT to fulfill the system's operations. BJTs were being studied as they show excel applications in analog or mixed signal ICs and Bipolar Complementary Metal-Oxide-Semiconductor (BiCMOS) circuits due to their high transconductance, linearity, low noise and excellent matching characteristics. Moreover, BJTs are widely used in amplifying, regulating or switching applications in space systems (Vergnault et al., 2000).

\section{MATERIALS AND MATHODS}

Test device descriptions: The DUT used in this test is NPN BJT (2N3904). This device is designed as a general purpose amplifier and switch. It is designed for low current and power, medium voltage and can operate at moderately high speeds. The useful dynamic range extends to $100 \mathrm{~mA}$ as a switch and to $100 \mathrm{MHz}$ as an amplifier.

${ }^{60}$ Co exposure: Exposures on the DUT were conducted with $\gamma$ rays, ${ }^{60} \mathrm{Co}$ source at RAYMINTEX Plant, Malaysian Nuclear Agency. Exposure of a semiconductor device to the $\gamma$ rays ${ }^{60} \mathrm{Co}$ is a popular and relatively inexpensive method of determining ionizing radiation damage susceptibility. ${ }^{60} \mathrm{Co}$ is a radioactive isotope of cobalt. Due to its short half life of 5.27 years ${ }^{60} \mathrm{Co}$ is not found in nature. It is produced artificially by neutron activation of ${ }^{59} \mathrm{Co} .{ }^{60} \mathrm{Co}$ decays by negative beta decay to the stable isotope ${ }^{60} \mathrm{Ni}$. The activated $\mathrm{Ni}$-atom emits two gamma rays with energies of 1.17 and $1.33 \mathrm{MeV}$.

Test setup: This radiation testing consisted of a step level multi parameter test. The DUT are electrically biased and are monitored in situ during exposure. This technique has numerous advantages such as increased measurement speed, reduced sensor introduction cost and increased spatial and temporal resolution. In line metrology in data acquisition system also produce a high accuracy system as it improved the process monitoring, reduce product variance and higher throughput.

The failure level for the device is reported in rad (Si), which is interpreted as the amount of energy deposited in the silicon-gate insulator and surface passivation regions by ionizing radiation. The diagram of the test setup was as shown in Fig. 1.

The information and status of the DUT were transmitted through the driver circuit based on an ADC circuit into the PC. This driver board served as the power supply to the DUT. Moreover, it was operated as a measuring tool to real time monitor the changes in the collector current $\left(\mathrm{I}_{\mathrm{C}}\right)$ and base current $\left(\mathrm{I}_{\mathrm{B}}\right)$ of the transistor. Cables used to connect this system should never be led to any serious distortion of the shape of signals or the degradation of reliability in data communication. The changes in the common-emitter current gain $\left(\mathrm{h}_{\mathrm{fe}}\right)$ was investigated at bias $\mathrm{I}_{\mathrm{C}}=5,10$ and $20 \mathrm{~mA}$ where:

$$
h_{\mathrm{fe}}=\frac{\mathrm{I}_{\mathrm{C}}}{\mathrm{I}_{\mathrm{B}}}
$$

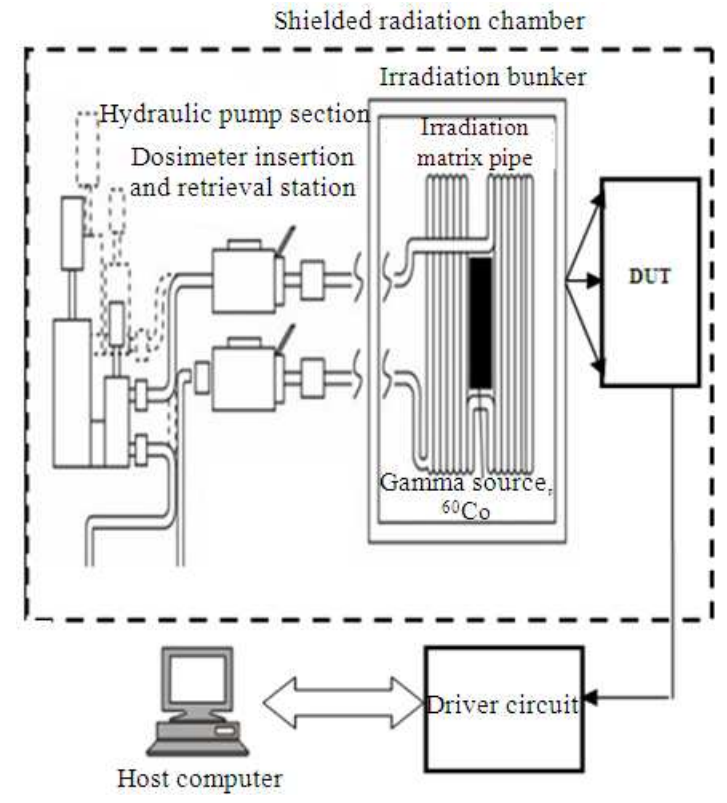

Fig. 1: ${ }^{60}$ Co Irradiation test setup 
Test condition: All test devices were verified to be functionally and parametrically working prior to irradiation. The control test was conducted for $72 \mathrm{~h}$ to verify that all the sample devices were well functioned within room temperature acceptance limits. The radiation test procedure was based on MIL-STD-883, method 1019-Ionizing Radiation (Total Dose) Test Procedure. Ambient temperature throughout the test was approximately $25 \pm 3^{\circ} \mathrm{C}$.

Fricke dosimeter was used to measure the absorbed dose at the fix selected point and the dose rate at that particular time was determined. Fricke dosimeter is a dose measuring device with a standard solution of $1 \mathrm{mM}$ $\mathrm{FeSO}_{4}$ in $0.8 \mathrm{~N} \mathrm{H}_{2} \mathrm{SO}_{4}$. The dose rate of the activity shows a variation at different time due to the half-life of Co-60. However, this variation was not significant for tests which were conducted at a same period. For example, the dose rate used to reach a total dose of $60 \mathrm{krad}$ (Si) in July 2009 was $5.0667 \mathrm{krad} \mathrm{sec}^{-1}$ and the duration taken was $3 \mathrm{~h} 17 \mathrm{~min} 22 \mathrm{sec}$. Hence, in order to compare the change in characteristics of the devices during the radiation state and non-radiation state. Similar tests were conducted at pre-irradiation with the same duration.

\section{RESULTS}

Exposures were done on ten NPN BJT (2N3904) devices at multi parameter and the change in characteristics were monitored in situ at $\mathrm{VCC}=10 \mathrm{~V}$. Figure 2 shows the percentage change in the $I_{B}$ at different bias $\mathrm{I}_{\mathrm{C}}$, given by:

$$
\% \Delta \mathrm{I}_{\mathrm{B}}=\frac{\mathrm{I}_{\mathrm{B} 1}-\mathrm{I}_{\mathrm{B} 0}}{\mathrm{I}_{\mathrm{B} 0}}
$$

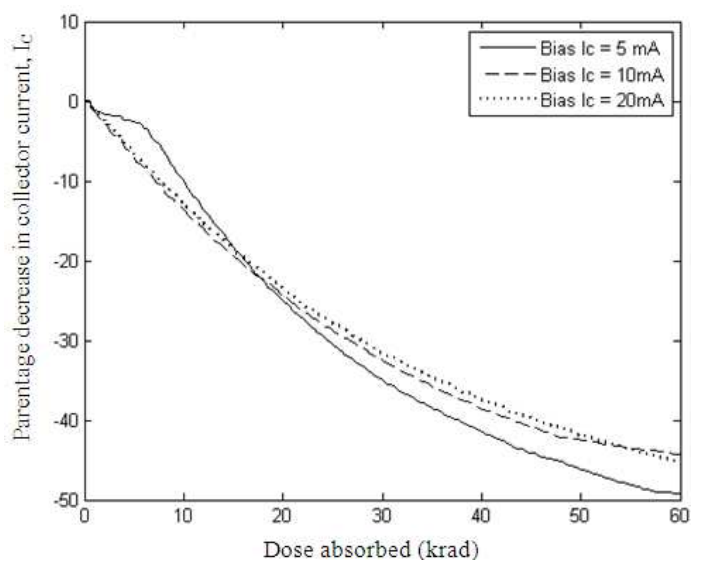

Fig. 2: Percentage increase in the base current, $I_{B}$ at different bias collector current, $\mathrm{I}_{\mathrm{C}}$
Figure 3 shows the percentage change in the $I_{C}$ at different bias $\mathrm{I}_{\mathrm{C}}$, given by:

$$
\% \Delta \mathrm{I}_{\mathrm{C}}=\frac{\mathrm{I}_{\mathrm{C} 1}-\mathrm{I}_{\mathrm{C} 0}}{\mathrm{I}_{\mathrm{C} 0}}
$$

Although $\mathrm{I}_{\mathrm{C}}$ was fixed at the beginning of the experiment, exposure to radiation, however, would change the value of this bias $\mathrm{I}_{\mathrm{C}}$.

Figure 4 shows the change in the $\frac{1}{\mathrm{~h}_{\mathrm{fe}}}$ at different bias $\mathrm{I}_{\mathrm{C}}$, given by:

$\Delta\left(\frac{1}{\mathrm{~h}_{\mathrm{fe}}}\right)=\frac{1}{\mathrm{~h}_{\mathrm{fe} 1}}-\frac{1}{\mathrm{~h}_{\mathrm{fe} 0}}$

Table 1-3 shows the statistics data for the data plot as that shown in Fig. 2-4 respectively.

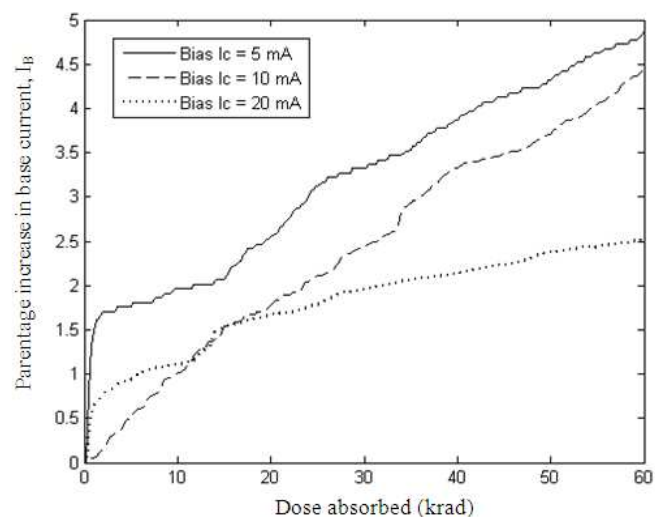

Fig. 3: Percentage decrease in the collector current, $I_{C}$ at different bias collector current, $\mathrm{I}_{\mathrm{C}}$

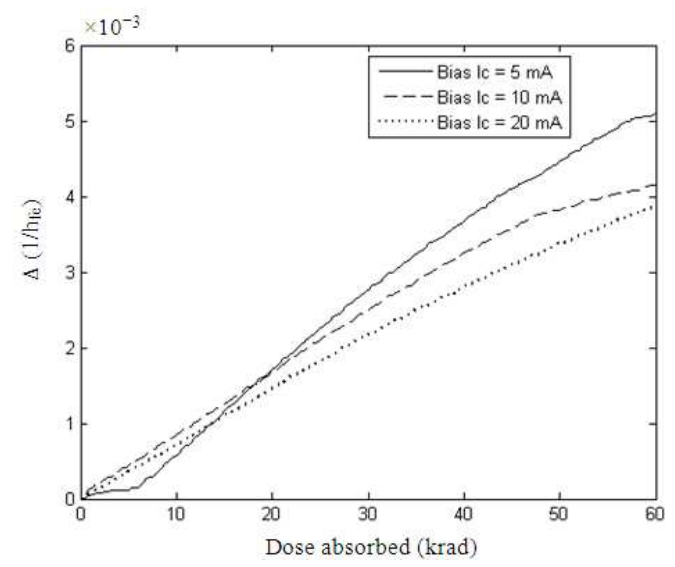

Fig. 4: Change in the $\frac{1}{\mathrm{~h}_{\mathrm{fe}}}$ at different bias collector current, $\mathrm{I}_{\mathrm{C}}$ 
Table 1: Statistics data for percentage change in base current, $\mathrm{I}_{\mathrm{B}}(\%)$ at different dose level for extraction from Fig. 2

\begin{tabular}{|c|c|c|c|}
\hline \multirow[b]{2}{*}{ Bias current, $\mathrm{I}_{\mathrm{C}}$} & \multicolumn{3}{|c|}{ Percentage change in base current, $\mathrm{I}_{\mathrm{B}}(\%)$} \\
\hline & Max value & Mean value & SD \\
\hline $5 \mathrm{~mA}$ & 4.879 & 3.212 & 1.0480 \\
\hline $10 \mathrm{~mA}$ & 4.438 & 2.427 & 1.2330 \\
\hline $20 \mathrm{~mA}$ & 2.518 & 1.816 & 0.5371 \\
\hline
\end{tabular}

Table 2: Statistics data for percentage change in collector current, $\mathrm{I}_{\mathrm{C}}$ (\%) at different dose level for extraction from Fig. 3

\begin{tabular}{llll}
\multicolumn{4}{c}{$(\%)$ at different dose level for extraction from Fig. 3 } \\
\hline & Percentage change in collector current, $\mathrm{I}_{\mathrm{C}}(\%)$ \\
Bias current, $\mathrm{I}_{\mathrm{C}}$ & Min value & Mean value & SD \\
\hline $5 \mathrm{~mA}$ & -49.24 & -30.51 & 15.63 \\
$10 \mathrm{~mA}$ & -44.31 & -29.22 & 13.96 \\
$20 \mathrm{~mA}$ & -45.25 & -28.55 & 13.00 \\
\hline
\end{tabular}

Table 3: Statistics data for $\Delta\left(\frac{1}{\mathrm{~h}_{\mathrm{fe}}}\right)$ at different dose level for extraction from Fig. 4

\begin{tabular}{llll}
\hline & $\Delta\left(\frac{1}{\mathrm{~h}_{\mathrm{fe}}}\right)$ & & \\
& - & & \\
& & & \\
Bias current, $\mathrm{I}_{\mathrm{C}}$ & Max value $\left(10^{-3}\right)$ & Mean value $\left(10^{-3}\right)$ & $\mathrm{SD}\left(10^{-3}\right)$ \\
\hline $5 \mathrm{~mA}$ & 5.091 & 2.633 & 1.631 \\
$10 \mathrm{~mA}$ & 4.154 & 2.383 & 1.267 \\
$20 \mathrm{~mA}$ & 3.861 & 2.085 & 1.142 \\
\hline
\end{tabular}

\section{DISCUSSION}

Ionizing radiation damage in the semiconductor devices is mainly caused by charges trapped on or near the surfaces of their insulating layers and interfaces. In bipolar devices, trapped charge at their surface layers produce inversion layers that expand the effective surface area, hence, modifying the surface potential at the $\mathrm{Si}_{-} \mathrm{SiO}_{2}$ interfaces.

From the results as shown in Fig. 2 and Table 1, it is found that the non-ideal $I_{B}$ increase with the total absorbed dose. This means that there is an increment in the surface state generation-recombination current. This can only happen when there is a depletion of $p$ base at the NPN BJTs under test which in turn leads to a reduction in the $\mathrm{I}_{\mathrm{C}}$ as shown in the Fig. 3 and Table 2. This means that there will be a decrement in the minority carrier life-time and hence decreasing the $\frac{1}{\mathrm{~h}_{\mathrm{fe}}}$ in the DUT. This can be shown in the Fig. 4 and Table 3.

From the data statistics obtained, it is found that the percentage change in $\mathrm{I}_{\mathrm{C}}$ and change in $\frac{1}{\mathrm{~h}_{\mathrm{fe}}}$ of the NPN BJTs is higher at lower bias $\mathrm{I}_{\mathrm{C}}$ of dose absorbed greater than $17 \mathrm{krad}$. This means that the spurious currents cause by the ionizing radiation will have a bigger impact at bipolar gain of low operating currents. It can be concluded that the gain degradation dispersions are larger for low bias current levels.

\section{CONCLUSION}

The degradation of the NPN BJT devices is depending on its operating current and also the Total Ionizing Dose (TID) absorbed. For bipolar transistor, decreased gain and increased leakage current are the most important parameters degraded by ionizing radiation.

\section{ACKNOWLEDGMENT}

The researchers are thankful to Science Fund 2007, MOSTI and Project No.: SCF0046-STS-2007, with title "Effects of Total Dose Irradiation on Semiconductor Devices".

\section{REFERENCES}

Aghara, S.K., 2003. Characterization of an in-core irradiator for testing of microelectronics in a mixed radiation environment. Ph.D. Dissertation, Texas University, Austin. http://repositories.tdl.org/tdl/handle/2152/423

Bhuva, B.H., 1987. The simulation of worst-case operating conditions for integrated circuits operating in a total dose environment. Ph.D. Dissertation, North Carolina State University.

Claeys, C. and E. Simoen, 2002. Radiation Effects in Advanced Semiconductor Materials and Devices. Springer-Verlag, Berlin Heidelberg Germany, ISBN: 13: 978-3540433934, pp: 401.

Guitierrez, A.O., 1999. Radiation effects on power mosfets. Ph.D. Dissertation, Rensselaer Polytechnic Institute, Troy, New York.

Li, X., K. Shen, C.M. Huang and L. Chu, 2007. A memory soft error measurement on production systems. Proceeding of the 2007 USENIX Annual Technical Conference, Santa Clara, CA., pp: 275-280.

Messenger, G.C. and M.S. Ash, 1992. The Effects of Radiation on Electronic System. Springer, Van Nostrand Reinhold, ISBN: 13: 978-0442239527, pp: 951.

Najim, J.M., 2009. Studying the different effects of gamma and X-ray irradiation on the electrical properties of silicon diode type 1 N1405. Int. J. Nanoelect. Mater., 2: 41-46.

Park, M., 2004. Total ionizing dose effects in power vertical double-diffused metal-oxidesemiconductor field effect transistors. University of New York at Buffalo.

Vergnault, E., R. Ecoffet, R. Millot, S. Duzellier and L. Guibert et al., 2000. Management of radiation issues for using commercial non-hardened parts on the INTEGRAL spectometer project. Proceeding of the IEEE Nuclear and Space Radiation Effects Conference Reno, July, 2000, IEEE Xlore Press, Nevada, $\quad$ pp: $68-79 . \quad$ DOI: 10.1109/REDW.2000.896272 